\title{
Individualised, flexible postnatal care: a feasibility study for a randomised controlled trial
}

Della A Forster ${ }^{1,2^{*}}$, Tracey L Savage ${ }^{1,2,7}$, Helen L McLachlan ${ }^{1,3}$, Lisa Gold ${ }^{4}$, Tanya Farrell ${ }^{2}$, Jo Rayner ${ }^{3}$, Jane Yelland ${ }^{5}$, Bree Rankin ${ }^{6}$ and Belinda Lovell ${ }^{2}$

\begin{abstract}
Background: Postnatal care in hospital is often provided using defined care pathways, with limited opportunity for more refined and individualised care. We explored whether a tertiary maternity service could provide flexible, individualised early postnatal care for women in a dynamic and timely manner, and if this approach was acceptable to women.
\end{abstract}

Methods: A feasibility study was designed to inform a future randomised controlled trial to evaluate an alternative approach to postnatal care. English-speaking women at low risk of medical complications were recruited around 26 weeks gestation to explore their willingness to participate in a study of a new, flexible model of care that involved antenatal planning for early postpartum discharge with additional home-based postnatal care. The earlier women were discharged from hospital, the more home-based visits they were eligible to receive. Program uptake was measured, women s views obtained by a postal survey sent at eight weeks postpartum and clinical data collected from medical records.

Results: Study uptake was 39\% (109/277 approached). Most women ( $n=103)$ completed a postnatal care plan during pregnancy; $17 \%$ planned to leave hospital within 12 hours of giving birth and 36\% planned to stay 48 hours. At eight weeks postpartum most women (90\%) were positive about the concept and $88 \%$ would opt for the same program again. Of the $28 \%$ who stayed in hospital for the length they had planned, less than half (43\%) received the appropriate number of home visits, and only $41 \%$ were given an option for the timing of the visit. Most (62\%) stayed in hospital longer than planned (probably due to clinical complications); 11\% stayed shorter than planned.

Conclusions: Women were very positive about individualised postnatal care planning that commenced during pregnancy. Given the hospital stay may be impacted by clinical factors, individualised care planning needs to continue into the postnatal period to take into account circumstances which cannot be planned for during pregnancy. However, individualised care planning during the postnatal period which incorporates a high level of flexibility may be challenging for organisations to manage and implement, and a randomised controlled trial of such an approach may not be feasible.

Keywords: Postnatal care, Domiciliary care, Individualised care, Early discharge, Postnatal preparation

\section{Background}

Whether postnatal care is provided in hospital or in the womans home, it is imperative that the care provided is of the highest standard and meets the needs of the individual [1] $p 21$.

\footnotetext{
* Correspondence: d.forster@latrobe.edu.au

'Judith Lumely Centre, La Trobe University, 215 Franklin St, Melbourne 3000,

Australia

${ }^{2}$ The Royal Women s Hospital, Locked Bag 300, Cnr Grattan St and Flemington Rd, Parkville 3052, Australia

Full list of author information is available at the end of the article
}

In Australia, increasing birth numbers, decreasing length of hospital postnatal stay and uncertainty regarding the optimal content and location of care provision are challenging issues in postnatal care. There is limited evidence about the impact of how care is provided in the early postnatal period - for example, impact(s) on the physical and/ or emotional health of the mother or the baby.

In Victoria, Australia, there has been increasing numbers of women giving birth [2] and a subsequent lack of physical space to provide hospital postnatal care for women. In response many hospitals have needed to discharge women

\section{Biomed Central}

? 2014 Forster et al.; licensee BioMed Central Ltd. This is an Open Access article distributed under the terms of the Creative Commons Attribution License (http://creativecommons.org/licenses/by/4.0), which permits unrestricted use, distribution, and reproduction in any medium, provided the original work is properly credited. The Creative Commons Public Domain Dedication waiver (http://creativecommons.org/publicdomain/zero/1.0/) applies to the data made available in this article, unless otherwise stated. 
much earlier than previously (with little evaluation of the effect of these changes), and the length of postnatal hospital stay has declined dramatically since the $1980 \mathrm{~s}$. In 2009, over one-third (39\%) of all women stayed in hospital two days or less, compared with 4\% in 1985 [2]. In 2009 10, the average length of stay for a public hospital birth episode was two days for an uncomplicated vaginal birth and four days for a caesarean section without major complications [3]. Following discharge from the public hospital system in Victoria, (where two thirds of all women have maternity care [3]) most new mothers receive the offer of at least one domiciliary midwife visit. The recently released Postnatal Care Program Guidelines for Victorian Health Services state that as a minimum requirement, following discharge, public health services should offer women at least one postnatal visit in her home, and that additional home visits [be] provided on the basis of individual clinical and psychosocial needs [1] p21. A statewide review of domiciliary care in Victoria found that overall, the median number of home visits for primiparous women was two (range one to four) and for multiparous women, one (range one to three) [4].

The Cochrane review of early postnatal discharge from hospital for healthy mothers and term infants concluded that early discharge does not appear to have adverse effects on breastfeeding or maternal depression when accompanied by a policy of offering women at least one nurse-midwife home visit post discharge [5]. However, the authors concluded that large well-designed trials of early discharge programs are needed, incorporating process evaluation to assess the uptake of co-interventions, and using standardised approaches to outcome assessment.

In terms of women s views of care, the component of maternity care which women consistently rate less favourably is postnatal care. This has been reported in Australia [6,7] and elsewhere [8-10]. Only half of the women participating in a state-wide survey of new mothers in Victoria in 2004 rated their postnatal care as very good, compared to $67 \%$ and $72 \%$ who rated their antenatal and intrapartum care respectively as very good [7]. Various factors were associated with satisfaction with care, and length of the hospital stay following birth was one of these; staying in hospital for one to two days was associated with less positive ratings of care compared with staying five days or more [7].

In 2006 we conducted focus groups in rural and metropolitan Victoria to gain more in-depth information on women s views of postnatal care and in particular to gain an understanding about their views on earlier postpartum discharge home. Fifty-two people participated in eight focus groups and four interviews [11]. This included eight pregnant women, of whom seven were pregnant with their first baby; 42 women who were in the postpartum period (some up to 12 months after the birth of their baby); and two partners. Women were generally concerned about the safety of their new baby, and lacked confidence in themselves as new mothers regarding their ability to care for their baby. There was a prevailing view that the physical presence and availability of professional support helped alleviate these concerns, and this was especially the case for women having a first baby. Women had anxieties and fears around early parenting and their changing role. Consistent with these views, many women were concerned about any moves to make the postnatal hospital stay shorter, especially for first time mothers. We concluded that any changes to care provision should be evaluated, that women s views should be taken into account, and that where possible providers should ensure that care is individualised to address each woman s/familys particular concerns [11].

Providers also have concerns about postnatal care provision. A Victorian state-wide review of hospital postnatal care based on the views of care providers found that there were a number of barriers to postnatal care provision including the busyness of postnatal wards, inadequate staffing numbers, and priority being given to other episodes of care $[12,13]$. The review highlighted a great diversity in the provision of postnatal care across the State in relation to models of care, staffing arrangements, and routine practices [13]. There was a strong sense among care providers that the provision of hospital based postnatal care is considered a lower priority than the other episodes of maternity care.

Given the context of maternity care in Victoria and elsewhere, it is important to consider how best to identify which women could receive more of their postnatal care at home, and what this care should involve. The provision of postnatal care at home following hospital discharge in the Australian context has had limited evaluation, although there are indications that women rate the care that they receive at home more highly than they do the care they receive in hospital [14-16]. A Victorian statewide review of home-based (domiciliary) care explored the structure and organisation of this care [4], but there is little data on the outcomes of home-based care, nor the views and experiences of women and care providers.

Although both the Victorian guidelines on postnatal care and the NICE guidelines on the routine care of postnatal women and their babies [17] suggest all care should be individualised, we identified no evidence regarding whether or not a more individualised approach to postnatal care is feasible or practicable from an organisational perspective, and whether the balance of care provided in hospital and at home can be optimised for each woman. In light of this, and considering that a number of Victorian maternity services were already moving towards very early postnatal discharge with little or no evaluation, we 
undertook a study to systematically pilot a new approach to postnatal care to provide a framework for further evaluation. The Postnatal individualised Care (PinC) Program was a feasibility study designed to inform a future randomised controlled trial which would evaluate an alternative approach to postnatal care. The aim was to explore the workability, costs and acceptability (to women and to care providers) of a new approach to early postnatal care, in particular the ability of a tertiary maternity service to provide flexible, individualised early postnatal care for women in a dynamic and timely manner.

Two aspects of this pilot ran simultaneously, a pilot of the intervention with women (the focus of this paper), along with focus groups and interviews with staff. This paper describes the alternative approach to early postnatal care we developed and presents data on whether women received the care we planned to be provided and what womens experiences were, as well as discussing the organisational factors that may have impacted on the implementation of the intervention. Staff views and costing data will be reported elsewhere.

\section{Methods}

\section{Study design}

A model of individualised postnatal care was developed and piloted. Eligible women were recruited to explore whether an individualised plan for postnatal care could be established in consultation with women at around 26 weeks gestation, and that it would be able to evolve to meet womens changing needs. This gestation was chosen as one of only two time points in pregnancy that all women attend the hospital for care (the other being the initial booking appointment), therefore considered the best option. No control group was included, as the aim was to determine the feasibility of the intervention within a metropolitan tertiary maternity hospital.

\section{Participants}

Women attending the Royal Womens Hospital in Melbourne (a large tertiary referral hospital with over 7,000 births per year) for pregnancy care as public patients were eligible. Women were excluded if they: were receiving birth centre care (associated with a short length of stay); had medical or obstetric risk factors that may have made them ineligible for short hospital stays; were less than 24 weeks gestation (although these women could be eligible later); were more than 30 weeks gestation (the intervention involved antenatal preparation, therefore women $>30$ weeks had inadequate time for antenatal preparation); were non-English speaking (NES); lived outside the hospital domiciliary area; were less than 16 years of age; or had significant social and psychological issues or risks. Women with planned or unplanned caesarean births were not excluded.

\section{Recruitment}

Brochures describing the program were available in all antenatal clinic waiting areas. Recruitment was conducted by a research midwife (TS). We aimed to approach all eligible women attending a selection of clinics that were chosen to be representative of all clinics held during the recruitment period, to ensure diversity in the sample. Women who agreed to participate provided written informed consent.

\section{Sample size}

We aimed to recruit up to 200 women, with no less than 100. These numbers were chosen not to show statistically significant differences in any outcomes, but to establish: the feasibility of undertaking this type of individualised, flexible care in a tertiary facility; the proportion of women willing to agree to participate in such a study; baseline data about likely cross-over in a model such as this; to explore issues in costing this type of care; and to identify the processes and education that would need to be put in place to implement a much larger version of this model.

\section{The intervention}

The underlying idea being tested was that women could trade time in hospital in the postnatal period for extra home visits following discharge, at a time and schedule that suited them. For example, if a woman went home within 12 hours of a normal birth she would be eligible for up to five home visits (as opposed to the more usual single visit) (Figure 1). In theory, the woman could choose to have a visit on the day of discharge, and even to have two visits on one day. That is, we aimed to test the ability of the organisation to provide postnatal care that was individualised and that was responsive to womens needs, provided in a timely manner, and in the context of the unpredictability of births. In addition, we wanted this to be a result of careful planning on the part of the woman and her family, not an ad hoc decision after the birth.

Women had a preliminary discussion on preparation for early discharge at the time of study recruitment (Table 1), to explore the concept of a flexible approach to early discharge and postnatal care at home, and to begin to consider what options would be most appropriate to their situation. They then had an additional appointment with the study midwife when attending for their 36 weeks gestation antenatal visit, to create their individualised plan of postnatal care. This antenatal planning for discharge was the key intervention, allowing the opportunity to ensure that the supports individual women might need at home were in place prior to labour and birth, well ahead of postnatal discharge wherever possible. Labour, birth and postnatal hospital care were provided as usual.

The postnatal plan, which detailed womens intended length of hospital stay and preference for scheduling of home visits, was documented on the PinC Program Individualised 


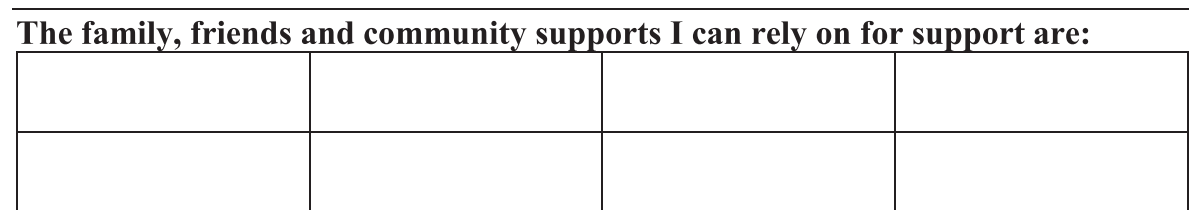

\begin{tabular}{|c|c|c|c|}
\hline \multicolumn{2}{|r|}{$\begin{array}{l}\text { After a Vaginal Birth I would like } \\
\text { to return home: }\end{array}$} & \multicolumn{2}{|c|}{$\begin{array}{l}\text { After a Caesarean Birth I would like } \\
\text { to return home: }\end{array}$} \\
\hline$\diamond$ & $\begin{array}{l}\text { The same day I give birth } \\
\text { Day } 0 \text { (6-12 hours) } \\
\text { eligible for up to } 5 \text { visits }\end{array}$ & 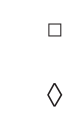 & $\begin{array}{l}\text { The day after the birth } \\
\text { Day } 1 \text { (up to } 24 \text { hours) } \\
\text { eligible for up to } 5 \text { visits }\end{array}$ \\
\hline$\diamond$ & $\begin{array}{l}\text { The day after the birth } \\
\text { Day } 1 \text { (12-24 hours) } \\
\text { eligible for up to } 4 \text { visits }\end{array}$ & $\diamond$ & $\begin{array}{l}\text { Two days after the birth } \\
\text { Day } 2 \text { ( } 24-48 \text { hours) } \\
\text { eligible for up to } 4 \text { visits }\end{array}$ \\
\hline$\diamond$ & $\begin{array}{l}\text { Two days after the birth } \\
\text { Day } 2 \text { ( } 24-48 \text { hours) } \\
\text { eligible for up to } 3 \text { visits }\end{array}$ & $\diamond$ & $\begin{array}{l}\text { Three days after the birth } \\
\text { Day } 3 \text { ( } 48-72 \text { hours) } \\
\text { eligible for up to } 3 \text { visits }\end{array}$ \\
\hline$\square$ & Other & $\diamond$ & Other \\
\hline
\end{tabular}

My preferred midwifery home visits:

Vaginal Birth
$1^{\text {st }}$ visit
$2^{\text {nd }}$ visit
$3^{\text {rd }}$ visit
$4^{\text {th }}$ visit
$5^{\text {th }}$ visit

Caesarean Birth
$1^{\text {st }}$ visit
$2^{\text {nd }}$ visit
$3^{\text {rd }}$ visit
$4^{\text {th }}$ visit
$5^{\text {th }}$ visit

This plan is flexible and can be altered at anytime to suit your preferences or medical needs, even after the birth. If your hospital stay is extended, the home visits you are eligible for will decrease by 1 for each extra day you stay in hospital. Regardless of the length of stay, you will be entitled to a minimum of 1-2 home visits.

\begin{tabular}{|l|l|}
\hline $\begin{array}{l}\square \text { The above plan reflects my preferences } \\
\text { prior to the birth }\end{array}$ & $\begin{array}{l}\square \text { The above plan reflects my } \\
\text { preferences after the birth } \\
\text { or } \\
\diamond \begin{array}{l}\text { I have modified this plan } \\
\text { tick diamonds and indicate home visits in red }\end{array} \\
\text { Signature }\end{array}$ \\
\hline day/month/year
\end{tabular}

Figure 1 PinC program individualised plan of postnatal care.

plan of postnatal care form (Figure 1), which was printed on bright pink paper and filed at the front of the woman s medical record. The plan could be modified at any time, including after the birth, allowing for a flexible length of stay for women. The aim was that at the time of discharge (as specified by the woman), care providers would arrange for the appropriate home visits to be provided at mutually agreeable times as per the agreed plan.

\section{Data collection and analysis}

Demographic data were collected by questionnaire, completed by women at recruitment. Additional data were collected from the postnatal plans completed at 36 weeks and by a postal questionnaire sent to women eight weeks postpartum. The questionnaire explored womens views and experiences of the new model, and included mainly structured questions, with opportunities for women to comment further. The questionnaire was based on survey instruments used previously $[18,19]$ and included questions specific to the project. A reminder letter was sent to all participants two weeks after the original mail-out and a phone call reminder after four weeks. Medical data pertaining to relevant readmissions were abstracted from the medical record a month after the birth. The number of women who were excluded and the proportion who consented to participate was documented to determine study uptake. 
Table 1 PinC individualised plan of postnatal care

\section{PinC Program pilot 36 week dialogue: a guide}

Introduction: This meeting is to discuss your preferred plan of care. You have had a couple of months to think about what you would like and I am interested to hear what you like to happen after you have your baby.

Assess personal support:

Have you had a chance to think about the supports you have at home? What family and friends do you intend to rely on? Who can help you with cooking, cleaning and shopping? Fill in details on plan

Community Support:

We will provide you with a direct phone number to speak with a midwife from your team once you go home. This number will be 24 hours a day for any question or concerns while you are at home. Your midwife may have already mentioned other community supports at your antenatal visits. Can you tell what community supports you are aware of? Fill in details on plan, prompt with relevant brochures if necessary

Length of Stay: Provided you and your baby are both healthy and medically cleared for discharge, how long do you think you would like to stay in hospital after the birth? Fill in details on plan

Home visits:

What is your preference for timing of home visits by midwives? Fill in details on plan ie: day 0 , day 1 , day 2 for home visits

Conclude visit:

Do you have any questions? Re-present program brochure given at time of recruitment. Participant to sign plan. For further questions or concerns, use the contact details on the brochure.

Data from questionnaires, medical records and postnatal individual plans were entered into a Microsoft Access database [20] and analysed using STATA version 8 [21]. Analysis for pre-coded responses was undertaken using descriptive statistics.

Womens responses (comments) to open-ended questions were analysed inductively and grouped into analytical descriptive categories [22]. Insights into womens experiences of the new model of care emerged from the comments they provided and direct quotes are used to illustrate the findings. As stated in the consent forms that were used, no information provided enables identification of individual women the three identifiers used for direct quotes are age, type of birth and whether it is a first baby. Given there are over 7,000 births per year at the study hospital this was considered sufficiently anonymising [23]. This aspect of the data analysis was done in accordance with the RATS guidelines [24]; that is, ensuring the Relevance of the research question, using an Appropriate method to collect the data, maintaining Transparent procedures (such as sampling, subject recruitment, ethics), and a Sound interpretive approach.

Ethical approval was provided by the Human Research Ethics Committee of La Trobe University (HEC 07 78) and the Royal Womens Hospital Research and Ethics Committees (Project 07 20).

\section{Results}

\section{Participants}

Women were recruited between September 2007 and January 2008 . Of the 1,687 potentially eligible women attending the 69 antenatal clinics attended by the research midwife, $82 \%$ were excluded after a brief review of the medical record, mainly due to gestation outside the eligible range (Figure 2). Of the 306 eligible women, 91\% (277) were approached and of those, 109 agreed to participate, representing 39\% of the eligible women approached. One participant withdrew later, decreasing the sample size to 108 , and another did not consent to medical record access, so data on birth outcomes and hospital care are presented for 107 women.

The majority of participants were married (67\%) or living with a partner $(30 \%)$ and had completed secondary school (91\%) (Table 2). Only 40\% of women were Australian born, however, 70\% indicated English was their first language. Seventy percent of women were having their first baby.

Women in this program were low risk at the time of recruitment, with no known medical problems that would prohibit early discharge, and $76 \%(81 / 107)$ did not have any complications recorded during pregnancy (Table 3 ). Of the 26 women with recorded pregnancy complications, most related to tests regarding fetal wellbeing. Births occurred between September 2007 and May 2008. The mean gestation at birth was 40 weeks (sd 2 weeks, range 2642 weeks). Three quarters of the women (79/107) had a vaginal birth. Thirty-four percent (36/107) had labour and birth complications documented in their medical record and 17\% (18/107) had postnatal complications documented.

All babies were liveborn and there were no neonatal deaths. The median five minute Apgar score was nine (range six to 10), and 15\% of all babies (15/107) required some form of resuscitation (Table 2). The mean birthweight was $3454 \mathrm{~g}$ (sd 544, range $805-4630 \mathrm{~g}$ ). Ten percent of babies $(11 / 107)$ were admitted to the special care nursery $(\mathrm{n}=8)$ or neonatal intensive care unit $(\mathrm{n}=3)$ during their initial hospital stay. Nearly all babies (98\%; 105/107) received at least some breast milk either directly from the breast or as expressed breast milk, although less than half (43\%) were discharged feeding only from the breast.

\section{Individual plans for postnatal length of stay (as decided at 36 weeks)}

Of the 108 participants, 103 women (95\%) completed a plan of postnatal care at 36 weeks gestation. Of the five women who did not complete the plan; two contacted the recruitment midwife prior to their appointment indicating they preferred standard care, one did not have Medicare cover $^{\mathrm{a}}$ and opted to decline all home visits, one relocated out of the hospital domiciliary visiting area, and another woman had a preterm birth at 26 weeks, soon after 


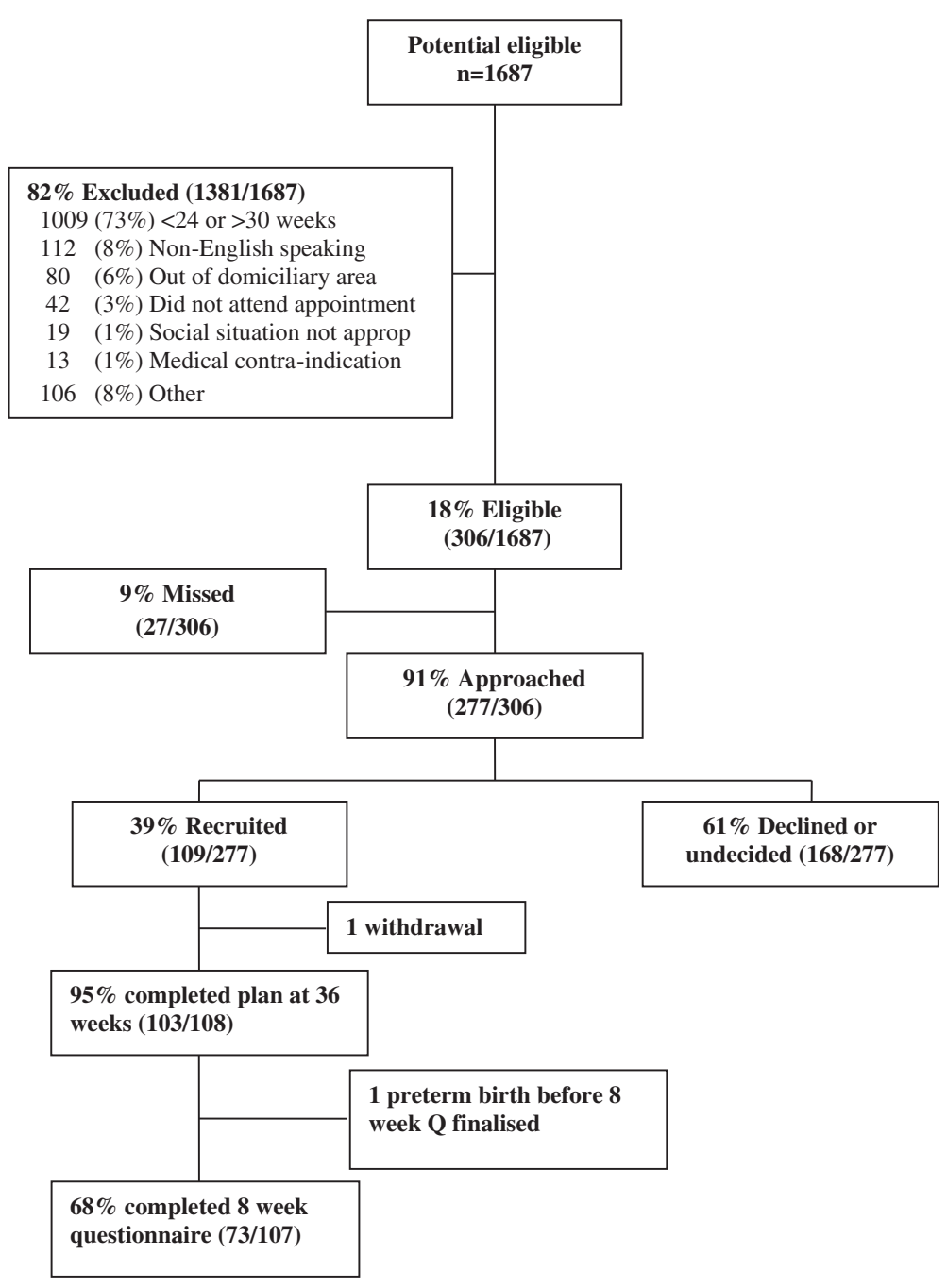

Figure 2 Participant recruitment.

recruitment. One woman gave birth (pre-term) before the eight week questionnaire was finalised. The remaining 107 women were sent questionnaires. Seventy-three of the 107 questionnaires were returned, a response fraction of $68 \%$.

Of the women who completed the plan regarding their postnatal stay following a possible vaginal birth, $17 \%$ planned to leave by 12 hours postpartum, and $36 \%$ planned to stay 24 to 48 hours. Less than one in four women $(23 \%, 24 / 103)$ chose to designate their length of stay in the case of a planned or unplanned caesarean birth. Of these 24 women, the majority preferred to stay three $(38 \%)$ or four nights $(38 \%)$. (NB: standard care in the public system at the time was 2.3 days following a vaginal birth and 3.9 days after a caesarean section [25] (Table 4).

In the questionnaire sent eight weeks after the birth women were asked if they considered that the concept of individualised postnatal care was introduced at an appropriate time during pregnancy, and 90\% (60/67) of respondents agreed:

I was starting to wonder about what would happen after the birth and the care I would receive. It was a great time to find out the options (ID1071, primiparous, 22 years, normal birth).

I hadn thought much about my hospital stay at this stage but discussing the program allowed me ample time to make a decision (ID1032, primiparous, 28 years, normal birth).

The majority (76\%; 54/71) of survey respondents indicated they felt actively involved in planning for the postnatal period during pregnancy . 
Table 2 Background characteristics of participants

\begin{tabular}{|c|c|c|}
\hline Characteristic & $\begin{array}{l}n \\
(n=1\end{array}$ & $\%$ \\
\hline Age (years), mean (sd) & 31.1 & 4.7 \\
\hline First baby $(n=107)$ & 75 & 70.1 \\
\hline \multicolumn{3}{|l|}{ Marital status: } \\
\hline Married & 72 & 66.7 \\
\hline Living with partner & 32 & 29.6 \\
\hline Have a partner but do not live together & 3 & 2.8 \\
\hline Single & 1 & 0.9 \\
\hline \multicolumn{3}{|l|}{ Highest education completed } \\
\hline Completed degree or higher & 57 & 52.8 \\
\hline Completed secondary school to year 12 & 42 & 38.9 \\
\hline Did not complete year 12 & 9 & 8.3 \\
\hline \multicolumn{3}{|l|}{ Country of birth $(n=103)$} \\
\hline Australia & 43 & 41.7 \\
\hline India & 10 & 9.7 \\
\hline New Zealand & 5 & 4.9 \\
\hline $\begin{array}{l}\text { Other* (includes } 30 \text { countries, each of which } \\
\text { represent }<5 \% \text { of participants) }\end{array}$ & 45 & 43.7 \\
\hline \multicolumn{3}{|l|}{ Length of time in Australia: $(n=56)$} \\
\hline Less than 5 years & 37 & 66.1 \\
\hline $5-10$ years & 3 & 5.4 \\
\hline More than 10 years & 16 & 28.6 \\
\hline English as a first language & 76 & 70.4 \\
\hline Smoked prior to pregnancy & 20 & 18.5 \\
\hline \multicolumn{3}{|l|}{ Income (pretax household income per week, \$AUD) } \\
\hline$<\$ 650$ & 12 & 11.1 \\
\hline$\$ 650-\$ 999$ & 18 & 16.7 \\
\hline$\$ 1000-\$ 1399$ & 23 & 21.3 \\
\hline$\$ 1400-\$ 1999$ & 26 & 24.1 \\
\hline$>\$ 2000$ & 25 & 23.2 \\
\hline Did not answer & 4 & 3.7 \\
\hline
\end{tabular}

\section{Length of postnatal stay}

The median length of stay after birth for women in the pilot was 2.8 days (range 4 hours to 9.3 days) (mean 2.6 days, sd 1.5). Thirty-one percent $(23 / 75)$ of the women having their first baby and 50\% (16/32) of those having a subsequent baby stayed less than 48 hours (Table 5). Considering vaginal births only, 48\% (38/79) were discharged less than 48 hours after the birth. Thirty-six percent of all women in the program were discharged less than 48 hours after the birth. This was twice the proportion of women being discharged prior to 48 hours in comparison to the general hospital population (18\% during March and April 2008), although women in this study were at low risk of complications, compared to the mixed-risk general hospital population.
Table 3 Maternal and infant clinical outcomes

\begin{tabular}{lll}
\hline Outcome & \multicolumn{2}{c}{$\begin{array}{l}\text { n } \\
\text { (n =107) }\end{array}$} \\
\hline Pregnancy complications (e.g. impaired fetal wellbeing) & 26 & 24.3 \\
Type of birth & & \\
Unassisted vaginal & 61 & 57.0 \\
Vacuum/forceps & 18 & 16.8 \\
Planned caesarean & 7 & 6.5 \\
Unplanned caesarean & 21 & 19.6 \\
Labour and birth complications* & 36 & 33.6 \\
Postpartum complications** & 18 & 16.8 \\
Birthweight (g) (mean, sd) & 3454 & $(544)$ \\
Liveborn & 107 & 100.0 \\
Admission to special care or neonatal intensive care & 14 & 13.1 \\
Apgar score at 5 minutes (median, range) & 9 & $(610)$ \\
Any breast milk feeding in hospital & 105 & 98.1 \\
\hline
\end{tabular}

*Postpartum haemorrhage (PPH) $n=26$; precipitate labour $n=3$; severe perineal trauma/hematoma $n=4$, shoulder dystocia $n=2$. **PPH $n=6$; hypogalactica $n=4$; endometritis $n=4$; urinary tract infection $n=2$.

\section{Planned versus actual length of hospital stay}

Although the antenatal plan included a section where women could alter their plan after the birth, most women (79/103) left this section blank. Medical record data were used to determine length of stay, and women $\mathrm{s}$ surveys used to ascertain if and why antenatal plans changed. Of those women who had a vaginal birth, $96 \%$ (76/79) had made an antenatal plan regarding their preferred length of stay after a vaginal birth. Of these, $62 \%$ (47/76) stayed longer than they planned, $11 \%(8 / 76)$ stayed a shorter time than they had planned, and $28 \%$ (21/76) stayed as planned. Of the women who had planned to stay 12 hours or less postpartum, 29\% (5/17) achieved this, and $35 \%(12 / 34)$ of those planning to go home between 12 and 24 hours did so. Ten of the women who made a plan regarding their preferred length of stay after a caesarean birth actually had a caesarean birth. Of these women, three stayed longer than planned, five stayed for their planned length and two stayed for less time than planned.

\section{Factors to explain postnatal length of stay that was longer or shorter than planned}

Of the women who stayed longer than planned following vaginal birth $(n=47)$, most extended stays appeared to be related to clinical complications. Postpartum haemorrhage (PPH) was the most common factor explaining the longer length of stay $(23 \% ; 11 / 47)$, with other possible factors including the woman being Group B Streptococcus positive $(n=10)$, having a third degree tear $(n=4)$, hematoma $(n=2)$, pre-eclampsia $(n=2)$, or incomplete placenta $(\mathrm{n}=2)$; or infant admitted to the 
Table 4 Plans for preferred length of postnatal stay made at 36 weeks gestation

n $\%$

Vaginal birth $(n=103)$

Leave the same day of the birth ( 612 hours)

One night stay (12 24 hours)

Two nights stay (24 48 hours)

$18 \quad 17$

$46 \quad 45$

$37 \quad 36$

Option was not applicable (having a planned caesarean birth) $2 \quad 2$

Caesarean birth $(n=24)$

One night stay (up to 32 hours)

Two nights stay ( up to 48 hours)

Three nights stay (48 72 hours)

Standard care ( $>72$ hours)

14

$5 \quad 21$

938

$9 \quad 38$

special care nursery $(n=2)$. However, from the medical records the exact reason(s) for extended length of stay was unclear and was not explicitly documented. The three women who stayed longer than planned after a caesarean birth also had at least one clinical complication that could explain the longer stay.

Although complications were often associated with stays longer-than-planned, this was not always the case. Nineteen percent $(7 / 36)$ of the women with complications of labour and/or birth had a length of stay less than 48 hours. Two women with a precipitate labour stayed between 12 and 24 hours while four women who had a PPH and one woman who had mild shoulder dystocia stayed between 24 and 48 hours.

When reflecting on their length of stay in the postpartum survey, $61 \%(39 / 64)$ of women indicated that they modified their plans after the birth of the baby, with $90 \%$ (35/39) staying longer than planned and 10\% (4/39) staying less time. Therefore many women did not achieve the length of stay they had planned during pregnancy (e.g. only $28 \%$ of those having a vaginal birth), and two thirds said that their plans changed after the birth of the baby. This needs to be taken into consideration in terms of whether women were happy with their length of stay, and whether they felt they had some control over what length of time they stayed in hospital.

\section{Home visits}

Overall, of those women who had a vaginal birth and who had made an antenatal plan regarding their preferred length of stay and home visits after a vaginal birth, $62 \%$ had the appropriate number of home visits as per their plan (Table 6). Regardless of whether the length of hospital stay was as planned, only one woman had more visits than planned, and $28 \%$ received less home visits than they should have according to their antenatal plan. For women who had a caesarean (who had made an antenatal plan for this outcome), 7/10 received the planned number of visits at home.

Only $37 \%(27 / 73)$ of respondents reported they were given an option for the number of postnatal home visits prior to being discharged home; however of these, 70\% $(19 / 27)$ reported receiving the number they requested. Similarly, only $41 \%$ of women (30/73) reported they were given an option for the timing of home visits. Notwithstanding this, women generally felt they received the right number of home visits (53/72; 74\%).

Forty-one women made comments in relation to the number or timing of visits, of whom $22 \%(\mathrm{n}=9)$ felt they had enough visits. Eight women indicated they would have preferred pre-arranged visit times.

It would have been more helpful if there was an estimate of time, rather than having to wait all day (ID 1093, multiparous, 34 years, normal birth).

[It] would be better to be informed of a time frame of visits, e.g. 9 12, to be able to plan when to sleep (ID 1050, primiparous, 30 years, normal birth).

The same number $(\mathrm{n}=8)$ indicated they would have liked more visits:

[The] midwives discharged me early as I was healthy and baby fine, but I would have liked one more visit just for reassurance (1051, primiparous, 28 years, normal birth).

Table 5 Postnatal length of stay by parity and method of birth

\begin{tabular}{|c|c|c|c|c|c|c|c|c|c|c|c|c|}
\hline & \multicolumn{2}{|c|}{$\leq 12$ hours } & \multicolumn{2}{|c|}{ 12-24 hours } & \multicolumn{2}{|c|}{ 24-48 hours } & \multicolumn{2}{|c|}{ 48-72 hours } & \multicolumn{2}{|c|}{$>72$ hours } & \multicolumn{2}{|c|}{$>96$ hours } \\
\hline & $\mathrm{n}$ & $\%$ & $\mathrm{n}$ & $\%$ & $n$ & $\%$ & $\mathrm{n}$ & $\%$ & $n$ & $\%$ & $n$ & $\%$ \\
\hline First baby $(n=75)$ & 3 & 4.0 & 4 & 5.3 & 16 & 21.3 & 16 & 21.3 & 22 & 29.3 & 14 & 18.7 \\
\hline Subsequent baby $(\mathrm{n}=32)$ & 2 & 6.3 & 8 & 25.0 & 6 & 18.8 & 8 & 25.0 & 5 & 15.6 & 9 & 9.4 \\
\hline Vaginal birth $(n=79)$ & 5 & 6.3 & 12 & 15.2 & 21 & 26.6 & 22 & 27.9 & 12 & 15.1 & 7 & 8.9 \\
\hline Caesarean birth $(n=28)$ & 0 & - & 0 & - & 1 & 3.6 & 2 & 7.1 & 15 & 53.6 & 10 & 35.7 \\
\hline All births $n=107$ & 5 & 4.7 & 12 & 11.1 & 22 & 20.6 & 24 & 22.4 & 27 & 25.3 & 17 & 15.9 \\
\hline
\end{tabular}


Table 6 Number of home visits compared to planned length of stay for vaginal births

\begin{tabular}{|c|c|c|c|c|c|c|c|c|}
\hline \multirow[t]{2}{*}{ Length of stay } & \multicolumn{2}{|c|}{$\begin{array}{l}\text { Home visits } \\
\text { as planned }\end{array}$} & \multicolumn{2}{|c|}{ More home visits } & \multicolumn{2}{|c|}{ Fewer home visits } & \multicolumn{2}{|c|}{$\begin{array}{l}\text { Unknown number } \\
\text { of visits }\end{array}$} \\
\hline & $\mathbf{n}$ & $\%$ & $\mathbf{n}$ & $\%$ & $\mathbf{n}$ & $\%$ & $\mathbf{n}$ & $\%$ \\
\hline As planned $(n=21)$ & 9 & 43 & 0 & 0 & 12 & 57 & 0 & 0 \\
\hline Longer than planned $(n=47)$ & 36 & 77 & 1 & 2 & 3 & 6 & 7 & 15 \\
\hline Shorter than planned $(n=8)$ & 2 & 25 & 0 & 0 & 6 & 75 & 0 & 0 \\
\hline Total (all women who had a vaginal birth and had plan $(n=76)$ ) & 47 & 62 & 1 & 1 & 21 & 28 & 7 & 9 \\
\hline
\end{tabular}

I would have liked two visits once at home as I felt pretty unsure about what I was doing (1082, primiparous, 31 years, normal birth).

\section{I would like another visit about a week later} (1061, primiparous, 30 years, normal birth).

Two women reported receiving less visits than agreed; one received a phone call instead of the last visit and the other indicated that the hospital did not provide the visits due to demand:

Two visits didn t occur because I was told we already have six visits booked for the next two days (1076, multiparous, 36 years, normal birth).

Only one woman reported she had too many visits:

I think midwives visiting every day is a bit much and unnecessary when its not the first child

(1094, multiparous, 28 years, normal birth).

\section{Women s views of the program}

Overall, women were supportive of the PinC program, with $88 \%(60 / 68)$ stating they would opt for the program in a future pregnancy and $87 \%(60 / 69)$ said they would recommend the program to family and friends.

\section{Positives}

One third of participants commented on the benefits of the program, including responses about being at home with their family, increased confidence with handling the baby at home and being able to get more rest.

I have the ability to breastfeed now and support of my partner at home during nights is fantastic. Id rather be at home with my family (ID 1105, primiparous, 35 years, forceps birth).

I would be able to come home to my own kind of dish [food]. A more familiar environment with less visitors in the room. And I would be able to use my own bathroom and toilet (ID 1041, multiparous, 35 years, normal birth).
Ten women liked the increased control and flexibility the program allowed, and these comments are typical.

We found the flexibility really worked for us. Knowing that we could stay or go home when ready, with support, allowed us to do what felt right at the time with confidence. The visits at home were invaluable (ID 1009, primiparous, 30 years, normal birth).

It was fantastic for me. It allowed me the ability to try and use my skills before being told/shown by someone else. Overall a very positive experience that has increased my confidence. The people involved in the program made it wonderful (ID 1083, primiparous, 29 years, normal birth).

It made me feel as though I had some control over my care (ID 1051, primiparous, 29 years, normal birth).

One said:

It empowers people to make their own decisions and not rely on the system as gospel (ID 1037, multiparous, 32 years, normal birth).

A number of the positive comments related to the domiciliary visits and the benefits of these.

We enjoyed having the midwife ourselves, one-onone for the extended period of time. We thought that really worked well (ID 1009, primiparous, 30 years, normal birth).

I don think you will receive more help in the hospital. PINC = one on one support in your own environment (ID 1104, primiparous, 30 years, normal birth).

A small number of participants $(n=7)$ would have preferred to spend less time in hospital.

\section{Negatives}

Five women s comments indicated a lack of staff awareness regarding the program. 
[I] didn t really get much info after the initial sign up. No one came to see me from the PinC program still don $t$ really know what it did in my particular case (ID 1039, primiparous, 36 years, normal birth).

After the birth, the Pinc program was not discussed with me at all - perhaps I should have been reminded that leaving early was an option and that I would still have phone support and home visits (ID 1061, primiparous, 30 years, normal birth).

Some women $(\mathrm{n}=14)$ considered the PinC program to be suitable only for women who already had children, or who were well supported or who had had vaginal births.

I suppose its not for everyone I [wouldn t] recommend it [to anyone] unless they are confident about the birth, probably for $2^{\text {nd }}$ baby (ID 1013, multiparous, 29 years, planned caesarean birth).

Only one woman wrote that she enjoyed the hospital stay and would not recommend the program saying,

I found the staff at the hospital knew more about ways to help me and advice to give me when I needed it. I felt relaxed and healed better in the hospital. Having someone do the cleaning, preparing meals etc. gave me more time to rest and focus on the baby. I got all the help without feeling invaded in my own home (ID 1012, multiparous, 29 years, normal birth).

One woman felt unable to make decisions on length of stay she might prefer given it was her first baby.

As it was my first baby, I didn $t$ know what to expect about my hospital stay so I found it difficult to guess how long I would want to stay, and what I would need help with after I went home (ID 1061, primiparous, 30 years, normal birth).

\section{Improving the PinC program}

Thirty-nine women suggested improvements that could be made to the program. Of these, 17 suggested a need for improved communication between multidisciplinary providers as well as between staff and women, and eight made comments relating to improving integration of the program into practice, with more educational support, information, referrals or phone support.

I found many midwives, doctors were unsure of the program while in hospital. I was ready to leave way before I was able to because I was chasing the midwife who did not know the process to follow. At most lead up appointments I had to tell them I was part of the program, they didnt seem to know (ID 1093, multiparous, 34 years, normal birth).

\section{Discussion}

Although it could be argued that all care should be individualised, the reality in large tertiary settings is that a set number of days or hours is broadly applied to the postpartum hospital stay, and there is little room for deviation except if clinical requirements mean a woman needs to stay in hospital longer. Similarly, while continuity of care models enhance individualised care, the vast majority of women do not have access to continuity of midwifery care at the current time, thus it is important to explore other ways of providing individualised postnatal care. The PinC program intervention was antenatal preparation for individualised postnatal care with a shorter length of hospital stay traded for increased domiciliary visits. We examined the feasibility and acceptability of this for women participating in the pilot program and whether such an intervention would be amenable to testing in a randomised controlled trial.

Nearly $40 \%$ of the women approached agreed to participate in the pilot. While reasons for non-participation were not specifically sought, some women said they were already planning to have early discharge but did not want to participate in research, and others (e.g. multiparous women desiring early discharge) said they did not need any extra home visits and did not see any benefit in participating. This may account for the relatively low proportion of multiparous women in the pilot, and it is possible that more than $39 \%$ would be agreeable to the idea of a shorter hospital stay with supported early discharge if it was not part of a research project. Many women seemed unaware that early discharge was an option in general.

During the discussion of the individualised PinC plan late in pregnancy, two-thirds of the women indicated they would like a stay of less than 24 hours after a vaginal birth. The discussion regarding the individual postnatal plans took place with a member of the research team. However, it is unknown if the women were also having discussions with midwives and doctors about postnatal discharge. These results show that women are willing to discuss plans for the postnatal period during pregnancy, and to consider planning for supportive early discharge.

Individualised postnatal plans were printed on bright pink paper and placed in the front of the medical record in an effort to alert staff. In many cases it seemed that staff were unfamiliar with the PinC program despite several education sessions and the involvement of the management team and key clinicians on the project steering committee. The section on the plans that was supposed to be completed in the postnatal period confirming the plans or modifying them was left blank in over $75 \%$ of 
cases, suggesting that plans were not referred to after the birth. This is supported by womens comments that staff appeared unaware of the plans. This level of missing data means we have no prospective data as to whether and how women changed their plans after birth; rather we have had to rely on their comments in the postal surveys. For the most part it is unknown whether changes were initiated by the women or by staff. Comments from the surveys indicated a high level of changes to plans in the early postnatal period, and a lack of staff knowledge about the program. It is likely that there were a combination of factors at play a possible lack of staff engagement and awareness of the PinC program and the impact of complications of labour, birth and/or the postnatal period (which were experienced by a large proportion of the women).

A relatively high proportion of women (28\%) received fewer home visits than planned. Of the 29 women (who had a vaginal birth) who had planned for a short hospital stay after a vaginal birth, and who stayed the same or less time than they planned, only 11 received the correct number of visits as per their postnatal plan (for example, a woman discharged home after one night should have received four visits as per the plan). The remaining 18 women all received fewer visits than the number indicated on the plan. There may have been issues around lack of staffing, limiting the ability to provide flexibility; a lack of knowledge of the program; or perhaps a philosophical view that women did not need so many postnatal home visits.

This apparent lack of staff engagement may have been largely due to a low awareness of the program. The number of women in the pilot represented a very small proportion (approximately 2\%) of overall births in the hospital. Although multiple education sessions were conducted, the staff attending these may not have provided care for women in the program. Brief information about the PinC program and a contact phone number for the project coordinator was located on a cover sheet for the postnatal plan, however, only three staff contacted the coordinator for more information or inquiries about the plan. Therefore it may have been the case that unless women identified themselves as part of the PinC program, staff were unaware. It is possible that if such an intervention was introduced on a larger scale there might be more engagement across the staff, with greater awareness of the program and increased adherence in terms of length of stay and number of postnatal home visits. Other explanations for the low proportion of women who achieved their plans could be that the staff did not agree with the PinC program, i.e. there was some cultural resistance to the change; that there were inadequate resources available to enact the plans (e.g. midwives, cars); or that women chose to accept the care as it was provided, rather than asking to have care as per their plans, or chose not to adhere to their plans. It may also be that for some women, especially first-time mothers, planning for the postnatal period during pregnancy might be quite challenging, given they are planning for something about which they know very little.

Despite the numerous studies that have found that women are less than satisfied with postnatal care and that care should be more individualised and flexible $[7,11,26]$ very few studies have implemented and evaluated new approaches to care. A before and after study conducted in Sydney, Australia, explored the impact of a multifaceted intervention which aimed to improve the content and quality of postnatal care. Strategies included one-to-one uninterrupted time between women and midwives, introduction of flexible breakfast arrangements, longer rest periods with minimal disturbance of women and where possible, provision of continuity of carer [26]. Although there were some positive changes (e.g. in strategies to improve rest for women), there were no significant differences in perceived quality of care, breastfeeding outcomes and maternal self-efficacy. The key strategy of one-to-one time was not consistently implemented and like this study, the authors concluded that there is the potential for individualised care to impact on outcomes, but established organisational systems and priorities are difficult to change. A statewide review of hospital postnatal care conducted in Victoria, Australia also found that organisational structures such as standard postnatal documentation and fixed length of stay, may inhibit rather than support individualised care for women after childbirth [13].

This project was undertaken during a time of major change. Relocation of the hospital to a new site took place a month after the last birth in the program, and a restructuring of maternity services was undertaken and a new primary midwifery model introduced around the same time. The focus for staff over this period was on preparation for these changes, which may have resulted in the PinC program receiving less attention than it may have at another time of implementation. As was occurring across Australia, the increased number of births had a direct impact on bed availability and stretched resources. The PinC program took place as the baby boom gathered momentum. Despite this, it may be that cultural factors were at play, such as midwives thinking a longer hospital stay is better for women. Although the women themselves seemed very open to the concept of the program, changing staff attitudes and hospital processes may be quite challenging. Involving midwives in the development of new interventions at the outset is an important consideration when midwives are the group required to ensure an intervention is implemented [27]. Understanding the PinC package from the midwives point of view is therefore critical, and the data from the focus groups and key stakeholder interviews (which will be reported elsewhere) will provide further insight into these issues. 


\section{Conclusion}

This feasibility study found that just over one third of low risk women were willing to participate in a study exploring individualised, planned early discharge followed by increased home-based postnatal care. Women were very positive about individualised postnatal care planning that commenced during pregnancy, however the proportion of women achieving their plans was much less than we had expected within a low risk population. The rate of obstetric intervention and postpartum complications was relatively high and staff perceptions of early discharge or lack of knowledge about the program may have contributed to the majority of women staying longer than planned.

Given these findings it is difficult to recommend that this particular approach would be achievable within the context of a randomised controlled trial, however similar approaches aiming to increase the individualised approach to postnatal care could be considered. Interventions based even more firmly within the current structures and with more potential buy-in from the clinical midwives providing home-based care warrant further investigation.

\section{Endnotes}

${ }^{\mathrm{a}}$ Medicare is Australias publicly funded universal health care system, operated by the government authority Medicare Australia. While Australia has reciprocal arrangements with some countries, citizens of most countries are not eligible for Medicare. Almost the entire population is covered by Medicare, with exceptions related to non-permanent residency status [28] Medicare Services [http://www.humanservices.gov.au/customer/subjects/medicare-services].

\section{Competing interests}

The authors declare that they have no competing interests.

\section{Authors contributions}

All authors were involved in study conceptualisation and implementation, including design of the intervention, study processes and data collection tools. DF and TS undertook data analysis. DF and TS drafted the manuscript initially and all authors were involved in revising subsequent drafts. All authors read and approved the final manuscript.

\section{Authors information}

Lovell Belinda, Maternity leave.

\section{Acknowledgements}

We gratefully acknowledge the Faculty Health Sciences, La Trobe University for funding the study; the women who participated in the study; and midwives who provided the care.

\footnotetext{
Author details

'Judith Lumely Centre, La Trobe University, 215 Franklin St, Melbourne 3000 Australia. ${ }^{2}$ The Royal Women s Hospital, Locked Bag 300, Cnr Grattan St and Flemington Rd, Parkville 3052, Australia. ${ }^{3}$ School of Nursing and Midwifery, La Trobe University, Melbourne Campus, Kingsbury Drive, Bundoora, Vic 3086, Australia. ${ }^{4}$ Deakin Health Economics, Deakin University, 221 Burwood Highway, Burwood 3125, Australia. ${ }^{5}$ Healthy Mothers Healthy Families Research Group, Murdoch Childrens Research Institute, 50 Flemington Road, Parkville, Victoria 3052, Australia. ${ }^{6}$ Drug Strategy Analysis Unit, Population 846 Health Division, Department of Health and Ageing, Canberra, Australia. ${ }^{7}$ Yale New Haven Hospital, 20 York Street, New Haven, CT 06510, USA.
}

Received: 14 April 2014 Accepted: 27 October 2014

Published online: 25 November 2014

\section{References}

1. Department of Health: Postnatal Care Program Guidelines for Victorian Health Services. In Melbourne, Victoria: Department of Health; 2012

2. Consultative Council on Obstetric and Paediatric Mortality and Morbidity: Annual Report for the Year 2009. In Melbourne: State Government of Victoria; 2012.

3. Department of Health: Victorian maternity services performance indicators: complete set for 2009 10. In Melbourne: State Government of Victoria; 2012.

4. McLachlan H, Forster D, McKay H, Ford R, Farrell T: Exploring the structure and organisation of home-based postnatal care in Victoria. Women and Birth 2011, 24(Suppl 1):S41.

5. Brown S, Small R, Faber B, Krastev A, Davis P: Early Postnatal Discharge from Hospital for Healthy Mothers and Term Infants (Review). In The Cochrane Library, Issue 2, Volume 2009. UK: John Wiley \& Sons, Ltd. Chichester; 2009.

6. Brown S, Darcy MA, Bruinsma F: Victorian Survey of Recent Mothers 2000. Early postnatal care. Melbourne: Centre for the Study of Mothers and Children s Health; 2001:1 42.

7. Brown SJ, Davey M-A, Bruinsma FJ: Women s views and experiences of postnatal hospital care in the Victorian survey of recent mothers 2000. Midwifery 2005, 21:109 126.

8. Newburn M, Bhavnani $\mathrm{V}$ : Left to your own devices: The posthatal care experiences of 1260 first-time mothers. London: NCT; 2010.

9. Redshaw M, Heikkila K: Delivered with care: a national survey of women's experiences of maternity care 2010. Oxford: National Perinatal Epidemiology Unit; 2010.

10. Rudman A, Waldenstr?m U: Critical views on postpartum care expressed by new mothers. BMC Health Serv Res 2007, 7(178):14.

11. Forster DA, McLachlan HL, Rayner J, Yelland J, Gold L, Rayner S: The early postnatal period: exploring women s views, expectations and experiences of care using focus groups in Victoria, Australia. BMC Pregnancy Childbirth 2008, 8:27.

12. Forster D, McLachlan H, Yelland J, Rayner J, Lumley J, Davey MA: Staffing in postnatal units: is it adequate for the provision of quality care? Findings from a state-wide review of postnatal care in Victoria, Australia. BMC Health Serv Res 2006, 6(83):14

13. McLachlan HL, Forster DA, Yelland J, Rayner J, Lumley J: Is the organisation and structure of hospital postnatal care a barrier to quality care? Findings from a statewide review in Victoria, Australia. Midwifery 2008, 24(3):358 370.

14. Biro M, Yelland J, Sutherland G, Brown S: Women s experience of domiciliary postnatal care in Victoria and South Australia: a population-based survey. Aust Health Rev 2012, 36(4):448 456.

15. Zadoroznyj M: Surveillance, support and risk in the postnatal period. Health Sociol Rev 2006, 15(4):353 363.

16. McLachlan H, Forster D, Davey M, Farrell T, Gold L, Waldenstr?m U, Biro M, Flood M, Albers L: A randomised controlled trial of caseload midwifery for women at low risk of medical complications (COSMOS): Women s satisfaction with care. Paediatrics 2012, 48(Supplement 1):41.

17. National Institute for Health and Clinical Excellence: Postnatal care (NICE quality standard 37). Manchester: National Institute for Health and Care Excellence; 2013

18. Waldenstrom U, Brown S, McLachlan H, Forster D, Brennecke S: Does team midwife care increase satisfaction with antenatal, intrapartum and postpartum care? a randomized controlled trial. Birth 2000, 27:156 167 .

19. McLachlan H, Forster D, Davey M-A, Lumley J, Farrell T, Oats J, Gold L, Waldenstrom U, Albers L, Biro M: COSMOS: COmparing Standard Maternity care with One-to-one midwifery Support: a randomised controlled trial. BMC Preg Childbirth 2008, 8(1):35.

20. Microsoft Corporation: Access 97 SR-2. Microsoft; 1997.

21. Stata Corporation: STATA 8.0 Statistics/Data Analysis. College Station, Texas: Stata Corporation; 2003.

22. Pope C, Ziebland S, Mays N: Analysing Qualitative Data. In Qualitative Research in Health Care. 3rd edition. Edited by Pope C, Mays N. Malden: Mass: Blackwell Publishing/BMJ Books; 2006:63 81. 
23. Hrynaszkiewicz I, Norton ML, Vickers AJ, Altman DG: Preparing raw clinical data for publication: guidance for journal editors, authors, and peer reviewers. Trials 2010, 11(9).

24. Clark J: How to Peer Review a Qualitative Manuscript. In Peer Review in Health Sciences. Secondth edition. Edited by Godlee F, Jefferson T. London: BMJ Books; 2003:219 235.

25. Department of Health: Births in Victoria 2007 and 2008. Melbourne: Consultative Council on Obstetric and Paediatric Mortality and Morbidity; 2010.

26. Schmied V, Cooke M, Gutwein R, Steinlein E, Homer C: An evaluation of strategies to improve the quality and content of hospital based postnatal care in a metropolitan Australian hospital. J Clin Nurs 2009, 18:1850 1861.

27. Goberna-Tricas J, Ban?s-Gim?nez M, Palacio-Tauste A, L-S S: Satisfaction with pregnancy and birth services: the quality of maternity care services as experienced by women. Midwifery 2011, 27(6):e231 e237.

28. Medicare services. [http://www.humanservices.gov.au/customer/subjects/ medicare-services]

doi:10.1186/s12913-014-0569-2

Cite this article as: Forster et al:: Individualised, flexible postnatal care: a feasibility study for a randomised controlled trial. BMC Health Services Research 2014 14:569.

\section{Submit your next manuscript to BioMed Central and take full advantage of:}

$\otimes$ Convenient online submission

$\otimes$ Thorough peer review

$\nabla$ No space constraints or color $\nabla$ gure charges

$\otimes I m m e d i a t e$ publication on acceptance

\Inclusion in PubMed, CAS, Scopus and Google Scholar

$\otimes$ Research which is freely available for redistribution 